\title{
Phenotypic characterization of Acinetobacter baumannii isolates from intensive care units at a tertiary-care hospital in Egypt
}

W. Nageeb, ${ }^{7}$ M. Kamel, ${ }^{7}$ S. Zakaria ${ }^{7}$ and L. Metwally'

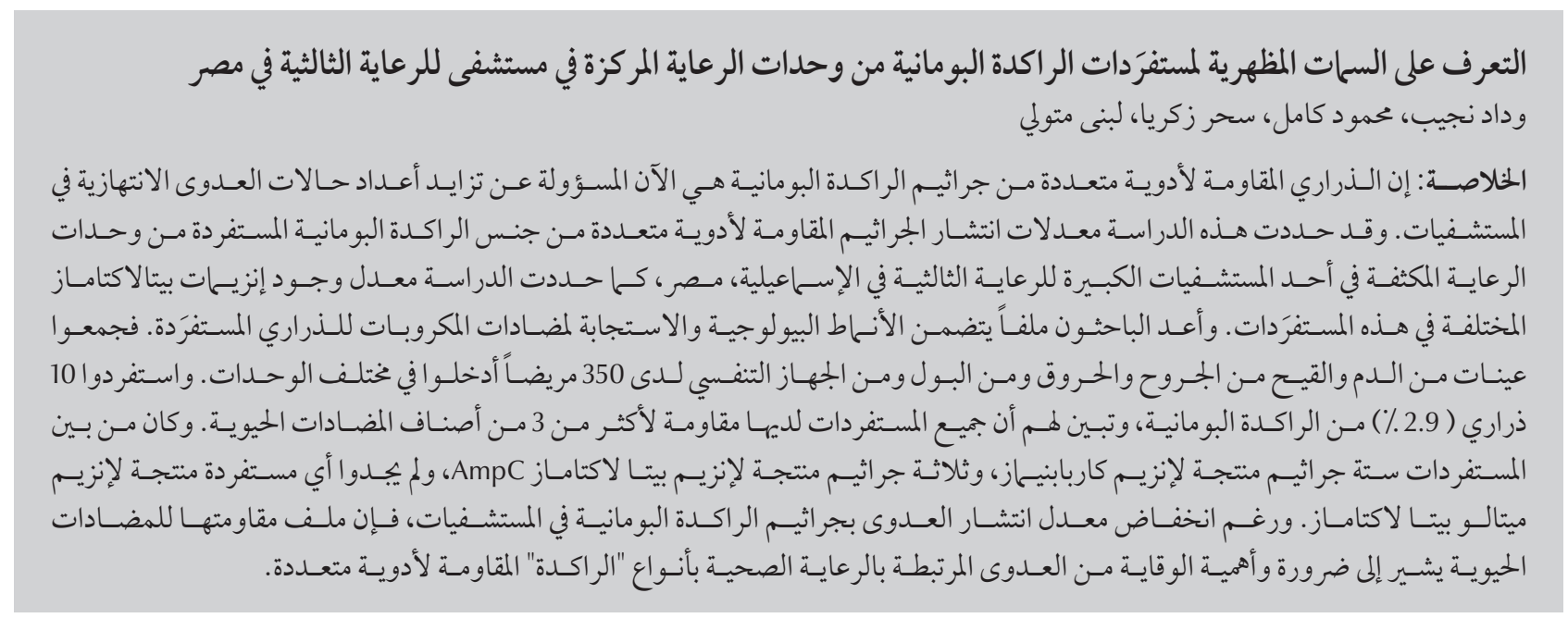

ABSTRACT Multi-drug resistant (MDR) strains of Acinetobacter baumannii are responsible for an increasing number of opportunistic infections in hospitals. This study determined the prevalence of MDR A. baumannii isolates from intensive care units in a large tertiary-care hospital in Ismailia, Egypt, and the occurrence of different beta-lactamases in these isolates. Biotyping and antimicrobial susceptibility profile was done for isolated strains. Respiratory, urine, burn wound and blood specimens were collected from 350 patients admitted to different units; 10 strains (2.9\%) of A. baumannii were isolated. All isolates showed resistance to more than 3 classes of antibiotics. Among the isolates, 6 isolates were carbapenemase producers, 2 were AmpC beta-lactamase producers and no isolates were metallo-betalactamase producers. Despite the low prevalence of $A$. baumannii infection in this hospital, the antibiotic resistance profile suggests that prevention of health-care-associated transmission of MDR Acinetobacter spp. infection is essential.

Caractérisation phénotypique des isolats d'Acinetobacter baumannii prélevés dans des unités de soins intensifs d'un hôpital de soins de santé tertiaires en Égypte

RÉSUMÉ Les souches d'Acinetobacterbaumannii multirésistantes sont aujourd'hui responsables de l'augmentation du nombre d'infections opportunistes dans les hôpitaux. La présente étude a déterminé la prévalence des isolats d'A. baumannii multirésistants prélevés dans des unités de soins intensifs d'un grand hôpital de soins de santé tertiaires à Ismaïlia (Égypte), et la fréquence de différentes bêta-lactamases dans ces isolats. Le biotypage et le profil de sensibilité aux antimicrobiens ont été établis pour les souches isolées. Des échantillons des voies respiratoires, d'urine, de blessures par brûlure et de sang ont été prélevés sur 350 patients admis dans différentes unités; 10 souches $(2,9 \%)$ d'A. baumannii ont été isolées. Tous les isolats présentaient une résistance à plus de trois classes d'antibiotiques. Parmi ces isolats, six produisaient des carbapénèmases, deux des bêta-lactamases AmpC mais aucun isolat ne produisait de métallo-bêta-lactamases. Malgré une faible prévalence de l'infection à $A$. baumannii dans cet hôpital, le profil de résistance aux antibiotiques laisse penser que la prévention de la transmission de l'infection à Acinetobacter spp. multirésistante associée aux soins de santé est essentielle.

'Department of Microbiology, Faculty of Medicine, Suez Canal University, Ismailia, Egypt (Correspondence to W. Nageeb: dr.wdnageeb@yahoo. com). 


\section{Introduction}

Of the newer pathogens encountered in health-care settings, Acinetobacter spp. are now recognized to play a considerable role in the colonization and infection of patients admitted to hospitals. Acinetobacter spp. have been implicated in a range of nosocomial infections, including bacteraemia, urinary tract infection (UTI), and secondary meningitis, but their predominant role is as agents of nosocomial pneumonia, particularly ventilator-associated pneumonia in patients confined to hospital intensive care units (ICUs) [1]. Outbreaks have been increasingly reported and most of those outbreaks are caused by multidrug resistant (MDR) strains of this organism. MDR strains of Acinetobacter spp. are now observed worldwide [2,3]. Many mechanisms act together to contribute to the problem of MDR including reduced access to microbial targets through loss of porin channels, possession of efflux pumps that are capable of actively removing a broad range of antimicrobial agents from the bacterial cell, and possession of a wide group of beta-lactamases that hydrolyse and give resistance to penicillins, cephalosporins and carbapenems $[4,5]$.

Regarding Egypt, there are no published data about the prevalence of $A$. baumannii in hospitals in our country. However, some reports are available from other developing countries. In the Islamic Republic of Iran, for example, a prevalence of $15 \%$ was reported [6], in India it was 9.5\% [7], while in Kuwait it was $22.1 \%$ [8]. In one study carried out in Saudi Arabia, A. baumannii was the most common isolated organism among Gram-negative bacteria, with a prevalence of $31.7 \%$ [9]. The current study aimed to phenotypically characterize A. baumannii isolated from different ICUs in our hospital and to describe the resistance profile of isolated strains. One of the objectives of our study was also to screen for the occurrence of these beta-lactamases and carbapenemases in our hospital as this is of importance in deciding the most appropriate therapeutic regimen for treatment of these beta-lactamase-resistant non-fermenting bacilli.

\section{Methods}

\section{Study setting}

This study was conducted over a period of 8 months, from June 2011 to February 2012, at the Suez Canal University Hospital, Ismailia, Egypt. Suez Canal University hospital is a tertiary-care teaching hospital in which the ICUs comprise an internal medicine unit (10 beds), a coronary care unit ( 7 beds), a hepatology unit ( 7 beds), a burns unit ( 10 beds) and a neonatal ICU (12 beds).

\section{Study sample}

A total of 350 specimens were collected randomly from 350 patients with clinical nosocomial infections admitted to different ICUs of the hospital. Only infections which occurred 48 hours following a patient's admission to hospital were evaluated. These included septicaemia ( 15 specimens), UTIs (120 specimens), respiratory infections (170 specimens) and burn wound infections (45 specimen). Only 1 specimen per patient was included in the study.

Ethical approval to perform the study was obtained from the ethics committee in the Faculty of Medicine, Suez Canal University and the management board of the hospital.

\section{Data collection}

Data collected from each patient included age, sex, length of hospital stay, use of invasive medical devices, receipt of antibiotics and the general surgical or medical problem necessitating admission.

Samples included urine (from both catheterized and non-catheterized patients), respiratory specimens (from both intubated and non-intubated patients), blood specimens and wound swab specimens. These were plated onto blood agar and MacConkey agar using the streaking method and then incubated aerobically at $37^{\circ} \mathrm{C}$ for 24-48 hours. Colonies which that were morphologically consistent with A. baumannii (i.e. oxidase-negative, catalase-positive, indole-negative, nonmotile, and glucose, lactose and sucrose non-fermenting when inoculated onto triple-sugar-iron agar) were then confirmed using an $\mathrm{API}^{\text {" }} 20 \mathrm{NE}$ kit (bioMérieux).

\section{Susceptibility testing}

Antibiotic susceptibility testing was determined using modified KirbyBauer method following the Clinical Laboratory Standards Institute guidelines [10]. E. coli ATCC 25922 was used as a quality control strain. The following antimicrobial agents were included in the panel: piperacillin $(100 \mu \mathrm{g})$, piperacillin/tazobactam $(100 / 10 \mu \mathrm{g})$, ampicillin/sulbactam $(20 / 10 \mu \mathrm{g})$, imipenem $(10 \mu \mathrm{g})$, meropenem $(10 \mu \mathrm{g})$, ceftazidime $(30 \mu \mathrm{g})$, cefotaxime $(30 \mu \mathrm{g})$, ceftriaxone $(30 \mu \mathrm{g})$, cefepime $(30 \mu \mathrm{g})$, ciprofloxacin $(5 \mu \mathrm{g})$, levofloxacin $(5 \mu \mathrm{g})$, gentamicin $(10 \mu \mathrm{g})$, amikacin $(30 \mu \mathrm{g})$, tobramycin $(10 \mu \mathrm{g})$, tetracycline $(30 \mu \mathrm{g})$ and trimethoprim/ sulfamethoxazole $(1.25 / 23.75 \mu \mathrm{g})$ (Oxoid).

With regards to tigecycline and colistin, there are considerable technical difficulties associated with susceptibility testing, as the disk diffusion method has been found to be inaccurate and not reproducible for colistin and tigecycline susceptibility testing of A. baumannii and this method is not recommended by the CLSI [11-13]. Agar dilution and broth microdilution are considered the gold standard susceptibility test methods for this organism. For determining susceptibility to polymyxins (polymyxin B and colistin) (Sigma-Aldrich), the minimum inhibitory concentration (MIC) was determined using the agar dilution method according to the European Committee on Antimicrobial 
Susceptibility Testing (EUCAST) [12]. The tested strains were deemed sensitive or resistant according to EUCAST breakpoints. For tigecycline (Wyeth Pharmaceuticals), both the disk diffusion and the agar dilution method were tested against the isolated strains. It is noteworthy that neither the CLSI [14] nor EUCAST [15] have any published MIC breakpoints for Acinetobacter spp. susceptibility testing against tigecycline. The interpretation of the zone diameters of tigecycline was done by using the US Food and Drug Administration breakpoints for susceptibility [16]. This showed a zone diameter of $\geq 19 \mathrm{~mm}$ as susceptible, $15-18 \mathrm{~mm}$ as intermediate, $\leq 14 \mathrm{~mm}$ as resistant. Most published studies to date have used a provisional breakpoint of $\leq 2 \mathrm{mg} / \mathrm{L}$ for determination of tigecycline susceptibility and resistance and this was used in our interpretation [17-19]. E. coli ATCC 25922 was used as a quality control strain for the agar dilution method used in MIC determination.

\section{Phenotypic detection of resistance mechanisms among A. baumannii isolates}

MDR A. baumannii isolates were defined as those resistant to more than 3 classes of antibiotic [20]. Stocks of 10 distinct MDR A. baumannii isolates, recovered from ICUs, were tested for the presence of different beta-lactamases by the following methods.

\section{Modified Hodge test}

An overnight culture suspension of $E$. coli 25922 adjusted to 1:10 dilution of $0.5 \mathrm{McF}$ arland standard was inoculated using a sterile cotton swab onto the surface of Muller-Hinton agar (MHA) (Hi-Media). After drying for 3-5 min, a $10 \mu \mathrm{g}$ meropenem disk (Oxoid) was placed at the centre of the test area. The test organism was streaked in a straight line from the edge of the disk to the edge of the plate. After an overnight incubation at $37^{\circ} \mathrm{C}$, the presence of a cloverleaf shaped zone of inhibition due to carbapenemase production by the test strain was considered as positive [21].

\section{EDTA disk synergy test}

An overnight liquid culture of the test isolate was adjusted to a turbidity of $0.5 \mathrm{McF}$ arland standard and spread on surface of MHA plate. A $10 \mu \mathrm{g}$ meropenem disk or $30 \mu \mathrm{g}$ ceftazidime disk was placed on the agar. A 0.5 M EDTA solution was prepared by dissolving 186.1 g of EDTA disodium salt (Reachem) in $1000 \mathrm{~mL}$ of distilled water. The $\mathrm{pH}$ was adjusted to 8.0 using $\mathrm{NaOH}$ (HiMedia) and sterilized by autoclaving. A blank disk (6 $\mathrm{mm}$ in diameter, Whatmann filter paper no. 1) was kept on the inner surface of the lid of the MHA plate and $10 \mu \mathrm{L}$ of $0.5 \mathrm{M}$ EDTA was added to it. The EDTA disk was then transferred to the surface of the agar and kept $10 \mathrm{~mm}$ edge-to-edge apart from the meropenem or ceftazidime disk. After incubating overnight at $37^{\circ}$ C, enhancement of zone of inhibition in the area between the meropenem and EDTA disk in comparison with the zone of inhibition on the far side of the drug was interpreted as positive for MBL production [22].

\section{AmpC disk test}

Tris-EDTA disks were prepared inhouse by applying $20 \mu \mathrm{L}$ of 1:1 mixture of saline and $100 \times$ tris-EDTA to sterile filter paper disks. The tris-EDTA disk was inoculated with heavy inoculums of the test organism prior to placing it on the plate. This was done in an empty Petri dish, using the wooden end of a sterile cotton swab to apply the organism. It was then inverted before placing it on the agar. This ensures optimal diffusion of the beta-lactamase into the agar. The susceptibility plate was inoculated with lawn of $0.5 \mathrm{McF}$ arland suspension of E. coli ATCC 25922. The cefoxitin disk was placed on the susceptibility plate. Then the TE disk with the test organism was placed close to $(1 \mathrm{~mm})$ but not touching the cefoxitin disk. After overnight incubation, the zone margin was examined for indentation or flattening which indicated evidence of AmpC beta-lactamase production [23].

\section{Results}

\section{Background data of patients}

This study included 350 patients with different nosocomial infections admitted to different ICUs of Suez Canal University Hospital during the period from June 2011 to February 2012. The study sample comprised 167 females (47.7\%) and 183 males (52.2\%). Their age ranged from 1 day to over 60 years, the mean age was 32.8 (standard deviation 21.7) years and most of them (37.7\%) were aged 35-60 years old (Table 1).

Half of our collected samples came from patients admitted to the general medical and surgical ICUs, while $22.9 \%$ were from patients admitted to the neonatal ICU, $12.9 \%$ from the burns ICU, $8.9 \%$ from the coronary care unit and $5.4 \%$ from the hepatology ICU. Most of the collected specimens were respiratory specimens (including both expectorated sputum and endotracheal aspirates) (170/350) (48.6\%),

\begin{tabular}{|c|c|c|}
\hline Age group & No. & $\%$ \\
\hline Neonate (0-28 days) & 55 & 15.7 \\
\hline Infant (1 month-2 years) & 25 & 7.1 \\
\hline Child (2-18 years) & 20 & 5.7 \\
\hline Young adult (18-35 years) & 107 & 30.6 \\
\hline Middle age (35-60 years) & 132 & 37.7 \\
\hline Elderly (> 60 years) & 11 & 3.1 \\
\hline
\end{tabular}




\begin{tabular}{lcc}
\hline $\begin{array}{l}\text { Table } 2 \text { Frequency of occurrence of bacterial species isolated from specimens } \\
\text { from intensive care unit patients }(\boldsymbol{n}=\mathbf{3 5 0})\end{array}$ & No. & $\%$ \\
\hline Organism & 60 & 17.4 \\
Klebsiella spp. & 35 & 10.0 \\
Escherichia coli & 125 & 35.7 \\
Pseudomonas spp. & 10 & 2.9 \\
Acinetobacter spp. & 15 & 4.3 \\
Proteus spp. & 21 & 6.0 \\
Other Gram -ve & 53 & 15.1 \\
Staphylococcus spp. & 31 & 8.9 \\
Other Gram +ve & &
\end{tabular}

followed by urine specimens (both catheterized and non-catheterized) (120/350) (34.3\%), burn wound swabs (45/350) (12.9\%) and blood specimens $(15 / 350)(4.3 \%)$.

\section{Organisms isolated}

The most frequently isolated organism from patients' samples was Pseudomonas spp. (125/350) (35.7\%), followed by Klebsiella spp. (60/350) (17.1\%). The least frequently isolated among the diagnosed organisms was Acinetobacter spp. (10/350) (2.9\%) (Table 2).

All 10 of the Acinetobacter spp. isolates in our study were confirmed to be A. baumannii by the API $20 \mathrm{NE}$ system. Isolation of $A$. baumannii was greatest from respiratory specimens $(50 \%$,
5/10), followed by blood specimens and burn wound swab specimens (both $20 \%, 2 / 10$ ), while 1 isolate came from a urine specimen.

Most of the A. baumannii isolates came from the general ICU and the neonatal ICU ( $40 \%$ for each, $4 / 10$ ). The remaining 2 isolates were from the burns unit. It was noted that $90 \%$ of patients with isolated A. baumannii $(9 / 10)$ had received antibiotics before isolation of the organism. In half of cases $(5 / 10)$ the patient stayed more than 15 days in hospital before the organism was isolated.

By the API 20NE system, 6 different biochemical profiles (biotypes) were identifiedin ourwards; biotype 0001073 was the most common, accounting for $30 \%(3 / 10)$ of isolates, followed by biotypes 0041073 and 4041473, each accounting for $20 \%(2 / 10)$. Biotypes 0001473, 4001073, 5041073 were each found in 10\% (1/10) of isolates.

\section{Antibiotic resistance of isolates}

Antibiotic susceptibility patterns of various isolates are shown in Table 3 , and the MIC results of polymyxins are shown in Table 4. All the 10 A. baumannii isolates were MDR (defined as resistance to more than 3 classes of antibiotics). All isolates were resistant to piperacillin, piperacillin/tazobactam, ampicillin/sulbactam, ceftazidime, cefepime, cefotaxime, ceftriaxone, imipenem, amikacin, ciprofloxacin and trimethoprim/sulfamethoxazole. Susceptibility to meropenem, gentamicin, tobramycin, tetracycline and levofloxacin enabled the definition of 5 phenotypic resistance patterns (A-E) which were equally distributed (20\% each). All isolates were sensitive to polymyxin B, although 2 isolates were resistant to polymyxin E (colistin), according to established CLSI breakpoints for MIC. When testing for tigecycline, the disk diffusion method and agar dilution for MIC showed variable results (Table 5) and according to the MIC, 2 isolates were tigecycline resistant.

\begin{tabular}{|c|c|c|c|c|c|c|c|c|c|c|c|c|c|c|c|c|c|c|}
\hline \multirow[t]{2}{*}{ Isolate } & \multirow[t]{2}{*}{ Biotype } & \multirow{2}{*}{$\begin{array}{l}\text { Antibiogram } \\
\text { group }\end{array}$} & \multicolumn{16}{|c|}{ Interpretation of disk diffusion test } \\
\hline & & & PRL & TPZ & SAM & CAZ & FEP & CTX & CRO & IPM & MEM & $\mathrm{CN}$ & TOB & AK & TE & CIP & LEV & SXT \\
\hline 1 & 0001073 । & A & $\mathrm{R}$ & $\mathrm{R}$ & $R$ & R & $\mathrm{R}$ & R & R & R & $\mathrm{R}$ & $S$ & $\mathrm{R}$ & R & I & $\mathrm{R}$ & $\mathrm{R}$ & $\mathrm{R}$ \\
\hline 2 & 4041473 III & B & $\mathrm{R}$ & R & $\mathrm{R}$ & $\mathrm{R}$ & R & R & $\mathrm{R}$ & $\mathrm{R}$ & $\mathrm{R}$ & $\mathbf{S}$ & I & $\mathrm{R}$ & $\mathrm{R}$ & $R$ & R & $\mathrm{R}$ \\
\hline 3 & 0041073 II & B & $R$ & $R$ & $R$ & $R$ & $\mathrm{R}$ & $R$ & $R$ & $R$ & $R$ & $S$ & I & $R$ & $\mathrm{R}$ & $R$ & $R$ & $\mathrm{R}$ \\
\hline 4 & 404147 III & C & $R$ & $R$ & $R$ & $R$ & $R$ & R & R & $R$ & $S$ & $\mathrm{R}$ & $R$ & $R$ & $S$ & $R$ & $S$ & $\mathrm{R}$ \\
\hline 5 & 0041073 II & C & $R$ & $R$ & $R$ & $R$ & $R$ & $R$ & $R$ & $R$ & $S$ & $\mathrm{R}$ & $R$ & $R$ & $S$ & $R$ & $S$ & $\mathrm{R}$ \\
\hline 6 & | & D & $R$ & $R$ & $R$ & $R$ & $R$ & $R$ & $R$ & $R$ & $R$ & $\mathrm{R}$ & $R$ & $R$ & I & $R$ & $R$ & $\mathrm{R}$ \\
\hline 7 & $5041073 \mathrm{VI}$ & D & $R$ & $R$ & $R$ & $R$ & $R$ & $R$ & $R$ & $R$ & $R$ & $\mathrm{R}$ & $R$ & $R$ & I & $R$ & $R$ & $\mathrm{R}$ \\
\hline 8 & | & $\mathrm{E}$ & $R$ & $R$ & $R$ & $R$ & $R$ & $R$ & $R$ & $R$ & $R$ & $\mathrm{R}$ & $R$ & $R$ & $\mathrm{R}$ & $R$ & $R$ & $\mathrm{R}$ \\
\hline 9 & $4001073 \mathrm{~V}$ & E & $R$ & $R$ & $R$ & $R$ & $R$ & $R$ & $R$ & $R$ & $R$ & $\mathrm{R}$ & $R$ & $R$ & $\mathrm{R}$ & $R$ & $R$ & $\mathrm{R}$ \\
\hline 10 & 0001473 IV & A & $R$ & $R$ & $R$ & $R$ & $R$ & $R$ & $R$ & $R$ & $R$ & $S$ & $R$ & $R$ & I & $R$ & $R$ & $\mathrm{R}$ \\
\hline
\end{tabular}

$R=$ resistant I = intermediate $S=$ sensitive.

$P R L=$ piperacillin; $T P Z=$ piperacillin/tazobactam; $S A M=$ ampicillin/sulbactam; $C A Z=$ ceftazidime; $F E P=$ cefepime; $C T X=c e f o t a x i m e ; C R O=c e f t r i a x o n e ; I M P=$ imipenem; $M E M=$ meropenem; $C N=$ gentamicin; $T O B=$ tobramycin; $A K=$ amikacin; $T E=$ tetracycline $; C I P=$ ciprofloxacin; $L E V=$ levofloxacin; $S X T=$ trimethoprim / sulfamethoxazole. 


\begin{tabular}{lcc}
\hline $\begin{array}{l}\text { Table 4 Minimum inhibitory concentration (MIC) of polymyxin B and of colistin } \\
\text { (polymyxin E) using disk diffusion method for the } 10 \text { Acinetobacter baumannii } \\
\text { isolates }\end{array}$ & \multicolumn{2}{c}{ MIC by disk diffusion test } \\
\hline Isolate & Polymyxin B (mg/L) & Colistin (mg/L) \\
& 0.5 & 64 \\
1 & 0.5 & 0.5 \\
2 & 0.25 & 0.5 \\
3 & 0.25 & 128 \\
4 & 0.25 & 0.5 \\
5 & 0.25 & 0.5 \\
6 & 0.125 & 0.5 \\
7 & 0.125 & 0.25 \\
8 & 0.125 & 0.5 \\
9 & 0.125 & 0.25 \\
\hline
\end{tabular}

For polymyxins, in Acinetobacter spp., MIC $\geq 4 \mathrm{mg} / \mathrm{L}$ is considered resistant, $M I C \leq 2$ is considered sensitive (Clinical and Laboratory Standards Institute guidelines [10])

Among the 10 imipenem-resistant isolates of A. baumannii, 6 isolates were carbapenemase producers (60\%) (positive by modified Hodge test), 2 isolates were beta-lactamase producers (20\%) (positive by AmpC test) and no isolates were MBL producers (all negative by EDTA disk synergy test) (Table 6). Two isolates were positive for both carbapenemase and AmpC beta-lactamases and another 4 isolates were positive for carbapenemase by modified Hodge test but were negative for MBL and AmpC beta-lactamases by EDTA disk synergy test and AmpC disk test respectively. Three isolates were not producing any of the 3 tested betalactamases by the 3 phenotypic tests used in our study.

\section{Discussion}

A. baumannii has emerged as a leading nosocomial pathogen, particularly in ICUs, where several outbreaks have been described $[1,24]$. The epidemic potential and the clinical severity of $A$. baumannii infections are primarily related to the propensity of this organism to develop resistance to a variety of antimicrobial agents, including broad-spectrum beta-lactams, aminoglycosides, fluoroquinolones and carbapenems [25]. This renders studies of epidemiology and antibiotic resistance necessary for prevention of further infection with these organisms.

In the current study the prevalence of A. baumannii was investigated from different ICU wards in our hospital by making phenotypic characterization using biotyping and antibiogram profiles to investigate the problem of MDR among A. baumannii isolates from these critical sites. During the study period, 350 clinical specimens collected from 350 patients with different nosocomial infections from different ICUs in Suez Canal University Hospital. Ten A. baumannii isolates $(2.9 \%)$ were identified. The results of the present study agree with those of Richards et al., who stated that A. baumannii represented $2.9 \%$ of all nosocomial infections [26]. Similarly, a low prevalence was reported by both Ruiz et al. and Lone et al., who found that A. baumannii represented $1.43 \%$

\begin{tabular}{|c|c|c|c|c|}
\hline Isolate & $\begin{array}{c}\text { MIC by disk } \\
\text { diffusion test }(\mathrm{mg} / \mathrm{L})\end{array}$ & Interpretation & $\begin{array}{l}\text { Zone diameter }(\mathrm{mm}) \\
\text { by agar dilution test }\end{array}$ & Interpretation \\
\hline 1 & 0.125 & S & 17 & I \\
\hline 2 & 32 & $\mathrm{R}$ & 8 & $\mathrm{R}$ \\
\hline 3 & 32 & R & 6 & $\mathrm{R}$ \\
\hline 4 & 0.25 & $S$ & 19 & S \\
\hline 5 & 1 & S & 11 & $\mathrm{R}$ \\
\hline 6 & 0.5 & $S$ & 12 & $\mathrm{R}$ \\
\hline 7 & 0.5 & S & 16 & 1 \\
\hline 8 & 0.5 & S & 17 & I \\
\hline 9 & 0.5 & $S$ & 13 & $R$ \\
\hline 10 & 0.5 & S & 20 & S \\
\hline
\end{tabular}

Breakpoint $\leq 2 \mathrm{mg} / \mathrm{L}$ was considered sensitive according to most published studies [17-19]. Neither CLSI nor EUCAST has any published MIC breakpoints for Acinetobacter spp. susceptibility testing against tigecycline).

Zone diameter $\geq 19 \mathrm{~mm}=$ sensitive, $15-18 \mathrm{~mm}=$ intermediate, $\leq 14 \mathrm{~mm}=$ resistant (US FDA susceptible breakpoints [16]).

$R=$ resistant,$I=$ intermediate,$S=$ sensitive.

$M I C=$ minimum inhibitory concentration; 


\begin{tabular}{lccc}
\hline \multicolumn{3}{l}{$\begin{array}{l}\text { Table } 6 \text { Phenotypic enzyme detection results for the 10 Acinetobacter baumannii } \\
\text { isolates by } 3 \text { different methods }\end{array}$} \\
\begin{tabular}{|cccc} 
Modified Hodge \\
test
\end{tabular} & $\begin{array}{c}\text { EDTA disk synergy } \\
\text { test }\end{array}$ & AmpC disk test \\
\hline 1 & $-v e$ & $-v e$ & $-v e$ \\
2 & $-v e$ & $-v e$ & $-v e$ \\
3 & $-v e$ & $-v e$ & $-v e$ \\
4 & $+v e$ & $-v e$ & $+v e$ \\
5 & $+v e$ & $-v e$ & $+v e$ \\
6 & $+v e$ & $-v e$ & $-v e$ \\
7 & $+v e$ & $-v e$ & $-v e$ \\
8 & $+v e$ & $-v e$ & $-v e$ \\
9 & $+v e$ & $-v e$ & $-v e$ \\
10 & $-v e$ & $-v e$ & $-v e$ \\
\hline
\end{tabular}

$-v e=$ negative $;+v e=$ positive .

and $4.8 \%$ of all nosocomial isolates respectively $[27,28]$.

The prevalence of $A$. baumannii in our study was much lower than the rates reported by other authors [29-31]. Kessaris et al. reported that the overall incidence of MDR A. baumannii positive cultures was 14.2\% [29]. Also Caricato et al. reported that Acinetobacter spp. accounted for $11 \%$ nosocomial infections [30]. Joshi et al. found that A. baumannii represented $9 \%$ of bacteriologically positive samples collected from a teaching hospital in India [31]. This can be explained by Siau et al. who showed a relative high frequency of A. baumannii infections in South-East Asian countries, and postulated that the hot and humid climates contributed to this high incidence [32].

In our study Acinetobacter spp. were isolated from respiratory, urine, blood and burn wound samples. Acinetobacter were most commonly isolated from specimens of patients with respiratory infections (5/10) and more rarely from urine samples $(1 / 10)$. These results agree with those of another 2 studies, which found that most of the Acinetobacter spp. isolates $(71.4 \%$ and $54 \%$ respectively) were from respiratory tract infection specimens [29,33]. In contrast, Villers et al. isolated Acinetobacter spp. most commonly from urine samples (31\%), followed by respiratory tract and wound samples $(26.7 \%$ and $17.8 \%$ respectively) [34]. These variations could be explained by the findings of Bergongne-Berezin, who stated that the predominant sites of Acinetobacter spp. nosocomial infection vary with time [35]. In early observations, UTIs predominated in ICUs. More recently, the incidence of UTI has decreased, possibly due to better care of urinary catheters, whereas the incidence of nosocomial pneumonia has increased significantly as reported by several recent surveys [35].

In our study, $A$. baumannii was the only Acinetobacter spp. encountered in clinical specimens and this supported the finding that infections by other Acinetobacter spp. are infrequent. Other studies found that among different Acinetobacter spp., A. baumannii was the most prevalent in clinical specimens and the one most often responsible for nosocomial infections $[28,31,36]$. As no single typing method has so far gained acceptance for typing Acinetobacter spp., and this area is still the subject of research, 2 approaches were used in our study: biotyping and antibiograms. By using those 2 phenotypic methods, our isolates were found to be distinct and non-duplicates [37].
Our isolates were shown to be $100 \%$ MDR (resistant to more than 3 groups of antibiotics) and 80\% extensively drug resistant (XDR) (also resistant to meropenem). Evans et al. in Pakistan found that MDR were $82.4 \%$ of his isolates and XDR were 65.0\% [38]. This differs from the results of Dent et al., who found that $72 \%$ of isolates were MDR and 58\% were XDR [39]. This difference may be due to the much larger total number of $A$. baumannii isolates in the latter study (247 isolates) than in our study (10 isolates).

Using the disk diffusion method for testing susceptibility to tigecycline showed that $5 / 10$ were resistant, $3 / 10$ were intermediate and 2/10 were sensitive. However, these results cannot be relied upon, as testing susceptibility against tigecycline using the disk diffusion method has been found to be inaccurate and not reproducible [11] and for this reason the agar dilution method for MIC determination was used (which is now considered the golden standard for testing susceptibility to tigecycline [11]) and this showed that $2 / 10$ were resistant and 8/10 were sensitive.

Beta-lactamase production is the most common mechanism of betalactam drug resistance in Gram-negative bacteria. Newer beta-lactamases that hydrolyse cephamycins, oxyimino and zwitterionic cephalosporins, monobactams, or carbapenems are of concern because they put a ceiling on the therapeutic options, lead to treatment failure and are increasing in prevalence $[40,41]$.

Currently, there is no CLSI recommended guidelines to detect AmpC beta-lactamases. In this study, we used AmpC disk testing and found that 20\% $(2 / 10)$ isolates were positive for the enzyme. This agrees with the results of Rodríguez-Martínez et al., who reported 29\% incidence of AmpC [42]. However, care is required in interpreting such results with isolates showing reduced carbapenem susceptibility since this 
may be due to other currently rare betalactamases (carbapenemases) capable of hydrolysing cefoxitin used in the test.

Carbapenems are often used as the antibiotics of last resort for treating infections due to MDR Gram-negative bacilli, because they are stable even in response to extended-spectrum and AmpC beta-lactamases. However, Gram-negative bacilli producing acquired MBLs (integron-encoded MBL and Verona integron-encoded MBL) have been increasingly reported in Asia and Europe [43]. Lee et al. have reported that the Hodge test can be used to screen carbapenemase-producing Gram-negative bacilli and that the imipenem-EDTA double-disk synergy test can distinguish MBL-producing from MBL-non-producing Gram-negative bacilli [44].

In our study we used the modified Hodge test for carpabenemases detection. It showed $60 \%$ of strains to be carbapenemases producers. This agrees with Andriamanantena et al., who detected carbapenemases in $86.8 \%$ of carbapenems-resistant isolates using the modified Hodge test [45]. On the other hand, Noyal et al. showed that among 46 resistant strains, only $14.3 \%$ was positive for carbapenemases by modified Hodge test and this was attributed to the presence of other mechanisms of resistance among the studied strains [46]. In our study the EDTA disk synergy test showed that no single strain was an MBL producer. This corresponds with the results obtained by Andriamanantena et al., who reported that among 53 tested strains, no isolates produced MBLs [45]. It contrasts with Gupta et al., who found that $7.5 \%$ of 200 Acinetobacter isolates were MBL producers [47]. This shows that MBL production is not an important mechanism of resistance among A. baumannii strains and that our tested strains may have been positive for carpabenemases due to the production of OXA-type carbapenemases. However, this needs to be confirmed by molecular methods.

This study has shown that tigecycline had good in vitro activity against the clinical isolates of the MDR A. baumannii, and that it may be considered as a promising therapeutic option for treatment. Nevertheless, tigecycline resistance was found among 2/10 isolates, as shown by the MIC values using the agar dilution method, and it is concerning to note that those isolates had not previously been exposed to the drug. So before starting treatment, the in vitro susceptibility of the isolates to tigecycline, and its MIC, should be assessed to prevent the development and the dissemination of resistance against one of the last available promising and safe therapeutic options that are available to clinicians to fight these bacteria.

This study also raises the importance of screening high-risk patients for carpabenemase producers in order to make a proper decision about treatment regimens. We recommend that proper infection control measures should be initiated to avoid dissemination of resistant carbapenemase-producing strains.

This study had a number of limitations, including those related to the considerable technical difficulties associated with tigecycline and colistin susceptibility testing. The disk diffusion method has been found to be inaccurate and not reproducible for colistin and tigecycline susceptibility testing of $A$. baumannii [11]. Agar dilution and broth microdilution are considered the gold standard susceptibility test methods for this organism. Both are cumbersome to perform and impractical to implement as routine tests in clinical laboratories $[11,14]$. Another limitation was that tigecycline MICs determined on Mueller-Hinton agar containing manganese at concentrations higher than $8 \mathrm{mg} / \mathrm{L}$ may produce falsely elevated MICs, a problem that may occur if testing is performed on standard media [48].

Our study suggests that continuous evaluation of antibiotic policy in hospitals should be done on a routine basis, to avoid irrational prescribing of antibiotics and to treat different infections according to their antibiotic susceptibility profile. With increasing reports of resistance to A. baumannii by the new glycylcyclines and polymyxins, together with the side-effects associated with these agents used as monotherapy, further research is recommended to study the possible effect of novel combination therapies as our last resort to treat such infections.

\section{Acknowledgements}

\section{Funding: None.}

Competing interests: None declared.

\section{References}

1. Guerrero DM et al. Acinetobacter baumannii-associated skin and soft tissue infections: recognizing a broadening spectrum of disease. Surgical Infections, 2010, 11:49-57.

2. Brusselaers N, Vogelaers D, Blot S. The rising problem of antimicrobial resistance in the intensive care unit. Annals of Intensive Care, 2011, 1:47-53.

3. Zhou $\mathrm{H}$ et al. Genomic analysis of the multidrug-resistant Acinetobacter baumannii strain MDR-ZJ06 widely spread in China. Antimicrobial Agents and Chemotherapy, 2011, 55:4506-4512.
4. Bonomo RA, Szabo D. Mechanisms of multidrug resistance in Acinetobacter species and Pseudomonas aeruginosa. Clinical Infectious Diseases, 2006, 43(Suppl. 2):S49-S56.

5. Rice LB. Challenges in identifying new antimicrobial agents effective for treating infections with Acinetobacter baumannii and Pseudomonas aeruginosa. Clinical Infectious Diseases, 2006, 43(Suppl. 2):S100-S105.

6. Shakibaie MR, Adeli S, Salehi MH. Antibiotic resistance patterns and extended-spectrum $\beta$-lactamase production among Acinetobacter spp. isolated from an intensive care Unit of a 
hospital in Kerman, Iran. Antimicrobial Resistance and Infection Control, 2012, 1:1-8.

7. Patwardhan $\mathrm{R}$ et al. A study on nosocomial pathogens in ICU with special reference to multiresistant Acinetobacter baumannii harbouring multiple plasmids. Indian Journal of Medical Research, 2008, 128:178-187.

8. AbdAllah $\mathrm{S}$ et al. Nosocomial infections and their risk factors at Mubarak Al-Kabeer hospital, Kuwait. Medical Journal of Cairo University, 2009, 78:123-131.

9. Al Johani SM et al. Prevalence of antimicrobial resistance among gram-negative isolates in an adult intensive care unit at a tertiary care center in Saudi Arabia. Annals of Saudi Medicine, 2010, 30:364-369.

10. Performance standards for antimicrobial susceptibility testing. Twenty-first informational supplement. Wayne, Pennsylvania, Clinical and Laboratory Standards Institute, 2011 (CLSI document M100-S21).

11. Gales AC, Reis AO, Jones RN. Contemporary assessment of antimicrobial susceptibility testing methods for polymyxin B and colistin: review of available interpretative criteria and quality control guidelines. Journal of Clinical Microbiology, 2001, 39:183-190.

12. European Committee for Antimicrobial Susceptibility Testing (EUCAST) of the European Society of Clinical Microbiology and Infectious Diseases (ESCMID). Determination of minimum inhibitory concentrations (MICs) of antibacterial agents by agar dilution. Clinical Microbiology and Infection, 2000, 6(9).

13. Jones RN, Anderegg TR, Swenson JM; Quality Control Working Group. Quality control guidelines for testing Gram-negative control strains with polymyxin B and colistin (polymyxin E) by standardized methods. Journal of Clinical Microbiology, 2005, 43:925-927.

14. Performance standards for antimicrobial susceptibility testing. Twenty-second informational supplement. Wayne, Pennsylvania Clinical and Laboratory Standards Institute, 2012 (CLSI document M100-S22)

15. Breakpoint tables for interpretation of MICs and zone diameters. Version 2.0. Valid from 2012-01-01. European Committee on Antimicrobial Susceptibility Testing [Internet] (http://www. eucast.org/fileadmin/src/media/PDFs/EUCAST_files/Breakpoint_tables/Breakpoint_table_v_2.0_120221.pdf, accessed 27 December 2013).

16. Tygacil (tigecycline) for injection [package insert]. Philadelphia, Pennsylvania, Wyeth Pharmaceuticals, 2005.

17. Navon-Venezia S, Leavitt A, Carmeli Y. High tigecycline resistance in multidrug-resistant Acinetobacter baumannii. Journal of Antimicrobial Chemotherapy, 2007, 59:772-774.

18. Thamlikitkul V, Tiengrim S, Tribuddharat C. Comment on: high tigecycline resistance in multidrug-resistant Acinetobacter baumannii. Journal of Antimicrobial Chemotherapy, 2007, 60:177-178.

19. Jones RN et al. Multicenter studies of tigecycline disk diffusion susceptibility results for Acinetobacter spp. Journal of Clinical Microbiology, 2007, 45:227-230.

20. Falagas ME, Koletsi PK, Bliziotis IA. The diversity of definitions of multidrug-resistant (MDR) and pandrug-resistant (PDR) Acinetobacter baumannii and Pseudomonas aeruginosa. Journal of Medical Microbiology, 2006, 55:1619-1629.

21. Anderson KF et al. Evaluation of methods to identify the Klebsiella pneumoniae carbapenemase in Enterobacteriaceae. Journal of Clinical Microbiology, 2007, 45:2723-2725.

22. Noyal MJ et al. Simple screening tests for detection of carbapenemases in clinical isolates of nonfermentative Gramnegative bacteria. Indian Journal of Medical Research, 2009, 129:707-712.
23. Black JA, Moland ES, Thomson KS. AmpC disk test for detection of plasmid-mediated AmpC beta-lactamases in Enterobacteriaceae lacking chromosomal AmpC beta-lactamases. Journal of Clinical Microbiology, 2005, 43:3110-3113.

24. Munoz-Price LS, Weinstein RA. Acinetobacter infection. New England Journal of Medicine, 2008, 358:1271-1281.

25. Abbo A et al. Multidrug-resistant Acinetobacter baumannii. Emerging Infectious Diseases, 2005, 11:22-29.

26. Richards M, Thursky K, Buising K. Epidemiology, prevalence, and sites of infections in intensive care units. Seminars in Respiratory and Critical Care Medicine, 2003, 24:3-22.

27. Ruiz J et al. Evolution of resistance among clinical isolates of Acinetobacter over a 6-year period. European Journal of Clinical Microbiology \& Infectious Diseases, 1999, 18:292-295.

28. Lone $\mathrm{R}$ et al. Nosocomial multi-drug-resistant Acinetobacter infections-clinical findings, risk factors and demographic characteristics. Bangladesh Journal of Medical Microbiology, 2009, 3:34-38.

29. Kessaris A et al. The incidence of infections caused by multidrug resistant Acinetobacter baumannii in an intensive care unit. Pneumon, 2006, 19:231-237.

30. Caricato A et al. Risk factors and outcome of Acinetobacter baumannii infection in severe trauma patients. Intensive Care Medicine, 2009, 35:1964-1969.

31. Joshi A et al. Multi-drug resistant Acinetobacter baumannii isolates from a teaching hospital. Journal of Infection and Chemotherapy, 2003, 9:187-190.

32. Siau $\mathrm{H}$ et al. The epidemiology of Acinetobacter infections in Hong Kong. Journal ofMedicalMicrobiology, 1996, 44:340-347.

33. Houang ET et al. Epidemiology and infection control implications of Acinetobacter spp. in Hong Kong. Journal of Clinical Microbiology, 2001, 39:228-234.

34. Villers D et al. Nosocomial Acinetobacter baumannii infections: microbiological and clinical epidemiology. Annals of Internal Medicine, 1998, 129:182-189.

35. Bergogne-Bérézin E. The increasing role of Acinetobacter species as nosocomial pathogens. Current Infectious Disease Reports, 2001, 3:440-444.

36. Basustaoglu AC et al. Epidemiological characterization of hospital-acquired Acinetobacter baumannii isolates from a 1500bed teaching hospital by phenotypic and genotypic methods. Journal of Hospital Infection, 2001, 47:246-247.

37. Lenie D. Typing Acinetobacter strains: applications and methods in Acinetobacter biology and pathogenesis. Infectious Agents and Pathogenesis, 2008, 85:104-109.

38. Evans BA et al. High prevalence of unrelated multidrug-resistant Acinetobacter baumannii isolates in Pakistani military hospitals. International Journal of Antimicrobial Agents, 2011, 37:580-581.

39. Dent L et al. Multi-drug resistant Acinetobacter baumannii: a descriptive study in a city hospital. BMC Infectious Diseases, 2010, 10:196-204.

40. Bradford PA. Extended-spectrum beta-lactamases in the 21st century: characterization, epidemiology, and detection of this important resistance threat. Clinical Microbiology Reviews, 2001, 14:933-951.

41. Bush K. New beta-lactamases in Gram-negative bacteria: diversity and impact on the selection of antimicrobial therapy. Clinical Infectious Diseases, 2001, 32:1085-1089.

42. Rodríguez-Martínez JM, Poirel L, Nordmann P. Genetic and functional variability of AmpC-type $\beta$-lactamases from Acinetobacter baumannii. Antimicrobial Agents and Chemotherapy, 2010, 54:4930-4933.

43. Livermore DM, Woodford N. Carbapenemases: a problem in waiting? Current Opinion in Microbiology, 2000, 3:489-495. 
44. Lee $\mathrm{K}$ et al. Modified Hodge and EDTA-disk synergy tests to screen metallo-beta-lactamase-producing strains of Pseudomonas and Acinetobacter species. Clinical Microbiology and Infection, 2001, 7:88-91.

45. Andriamanantena $T$ et al. Dessimination of multidrug resistant Acinetobacter baumannii in various hospitals of Antananarivo Madagascar. Annals of Clinical Microbiology and Antimicrobials, 2010, 9:1-6.

46. Noyal MJ et al. Simple screening tests for detection of carbapenemases in clinical isolates of nonfermentative Gramnegative bacteria. Indian Journal of Medical Research, 2009, 129:707-712.
47. Gupta V, Datta P, Chander J. Prevalence of metallo-beta lactamase (MBL) producing Pseudomonas spp. and Acinetobacter spp. in a tertiary care hospital in India. Journal of Infection, 2006, 52:311-314.

48. Veenemans J et al. Effect of manganese in test media on in vitro bacterial susceptibility to tigecycline of Enterobacteriaceae and Acinetobacter baumannii to tigecycline. Journal of Clinical Microbiology, 2012, 50:3077-3079. 